PII: S0031-3203(98)00058-2

\title{
ON-LINE SIGNATURE VERIFICATION BASED ON LOGARITHMIC SPECTRUM
}

\author{
QUEN-ZONG WU, ${ }^{* \dagger}{ }^{\dagger}$ SUH-YIN LEE ${ }^{\ddagger}$ and I-CHANG JOU \\ ${ }^{\dagger}$ Chunghwa Telecommunication Laboratories, P.O. Box 71, Chung-Li, Taiwan, R.O.C. \\ ${ }^{\ddagger}$ Department of Computer Science and Information Engineering, National Chiao Tung University, \\ Hsinchu, Taiwan, R.O.C. \\ ${ }^{\S}$ Department of Electrical Engineering, Chang Gung College of Medicine and Technology, Tao-Yuan, \\ Taiwan, R.O.C.
}

(Received 10 September 1997: in revised form 2 April 1998)

\begin{abstract}
In this paper, an on-line signature verification scheme based on similarity measurement of logarithmic spectrum is proposed. The principal components of the logarithmic spectrum of each signature are extracted. We then compute the similarity of logarithmic spectrum between input signature and the reference template. By comparing the similarity of logarithmic spectrum with the verification threshold, we can determine the authenticity of the input signature. Based on the experimentation, the rates of false rejection errors and false acceptance errors are as low as 1.4 and $2.8 \%$, respectively. This demonstrates the effectiveness of the proposed scheme. (C) 1998 Published by Elsevier Science Ltd on behalf of the Pattern Recognition Society. All rights reserved.
\end{abstract}

Signature verification Principal component

Similarity measurement

Logarithmic spectrum

Scatter matrix

\section{INTRODUCTION}

As computers are causing another revolution on the life styles of human beings, processes and schedules are affected or even completely reorganized. Office automation is one example. By widely applying computers and peripherals, such as printers, scanners and digital tablets, we are entering the era of intelligent input/output and electronic documentation.

Signature verification provides a way to determine the validity and authority of documents. It could also be applied to security systems to protect critical data or information from unauthorized use. Therefore, signature verification is of great importance in electronic documentation. Its applications include banking, credit-card authorization and personal identification.

In most cases, signatures are verified through visual examination. However, to tell genuine signatures from forged ones is not easy. For instance, in a commercial transaction, comparing two signatures is a challenge for clerks.

Recently, many methods ${ }^{(1)}$ have been developed for computer-based signature verification, especially for on-line signature verification. Figure 1 shows the basic modules for on-line signature verification. Static features, such as coordinates, as well as dynamic features, including writing velocity and acceleration, are useful characteristics for on-line signature verification.

*Author to whom correspondence should be addressed.
Other issues to be considered in a verification system are similarity measurement, a learning scheme on the signatures and criteria for forgery determination. ${ }^{(1)}$

Static analysis measures the similarity of the outlooking shape between input and reference signatures, while dynamic analysis measures the similarity of signature dynamics between input and reference signatures. Signature dynamics is the style of writing speed or the changing rate of coordinates within the signature. The dynamics of signing is less easily to imitate than the outlooking shape of the signature because the dynamics of signing is difficult to observe by human eyes. Velocity, acceleration and spectrum can be used to characterize the changing rate of coordinates within the signature. Figure 2 shows a Chinese signature and its $x$ and $y$ coordinates.

The changing rate among the sample points of a time sequence can be presented by transforming the time sequence to frequency domain. On-line signatures are time sequences of coordinates. Hence, we can examine the changing rate of the coordinates among the sample points of a signature by transforming the sequence of coordinates to frequency domain. Lam and Kamins ${ }^{(2)}$ used the largest 15 harmonics derived from fast Fourier transform (FFT) as the features of the signatures. Because the exponential operations are imbedded in the computation of FFT, the waveform of the FFT may be sharp or make large difference due to very little variation in input signal. Hence, if spectrums are not properly aligned, then the variation of distance or similarity among spectrums would be large. Instead, we can compute the distance 
or similarity between two logarithmic spectrum. ${ }^{(3-5)}$ Due to the logarithmic operations on the spectrum, the waveform of the logarithmic spectrum is much smoother than that of the spectrum. The differences of corresponding coefficients between two spectrums are

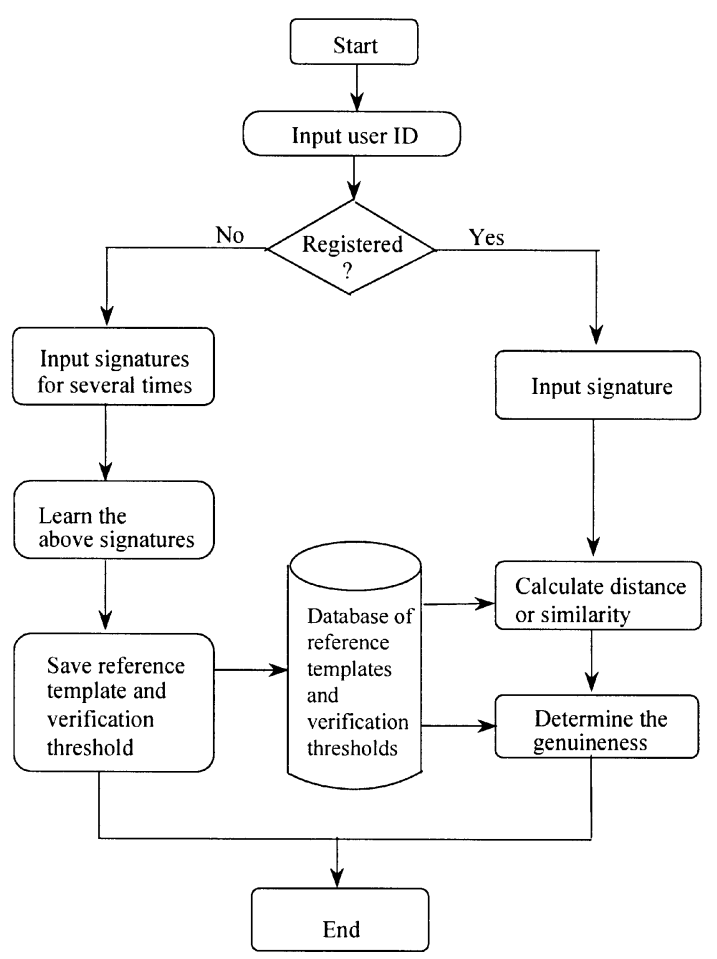

Fig. 1. Basic modules for a signature verification system. scaled down and might not be extraordinary large or extraordinary small. Thus, the similarity or distance of the changing rate of coordinates between two signatures can be characterized by their similarity of the coefficients of logarithmic spectrum. Figures 3 and 4 show the spectrum and the logarithmic spectrum of a signature whose $x$ - and $y$-coordinates are normalized to 1 .

Cepstral analysis is widely used in the area of speech processing ${ }^{(3-5)}$ due to its capability to well represent speech waveforms and characteristics with a small number of parameters. The cepstrum of a waveform $c$ is defined as the inverse Fourier transform of the logarithmic spectrum IFFT $(\log |F F T(c)|)$. Wu et $a l^{(6,7)}$ used LPC (Linear Predictor Coding) cepstrum $^{(3-5)}$ as the features of the signatures for on-line signature verification based on neural networks.

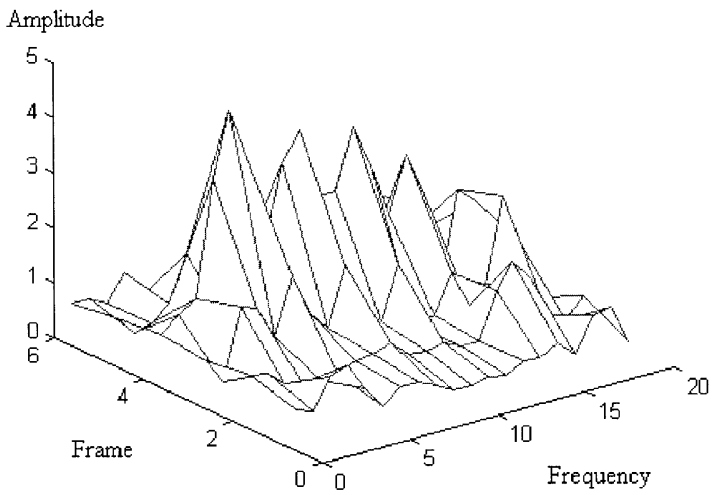

Fig. 3. The spectrum of a signature.
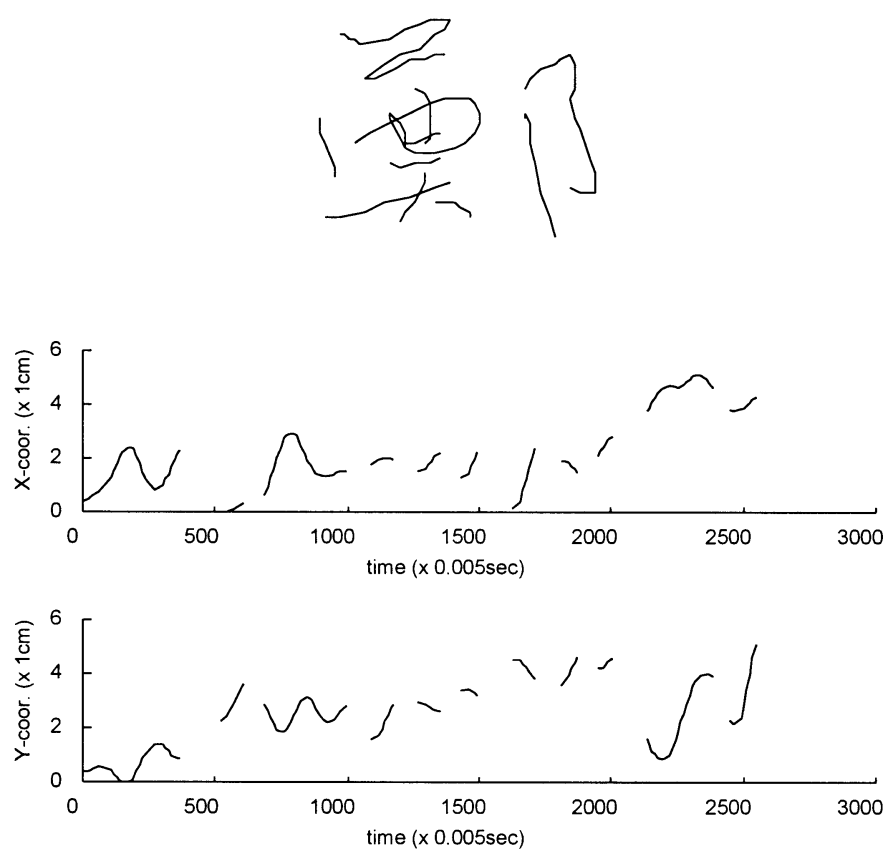

Fig. 2. A Chinese signature and its $x$ and $y$ coordinates. 


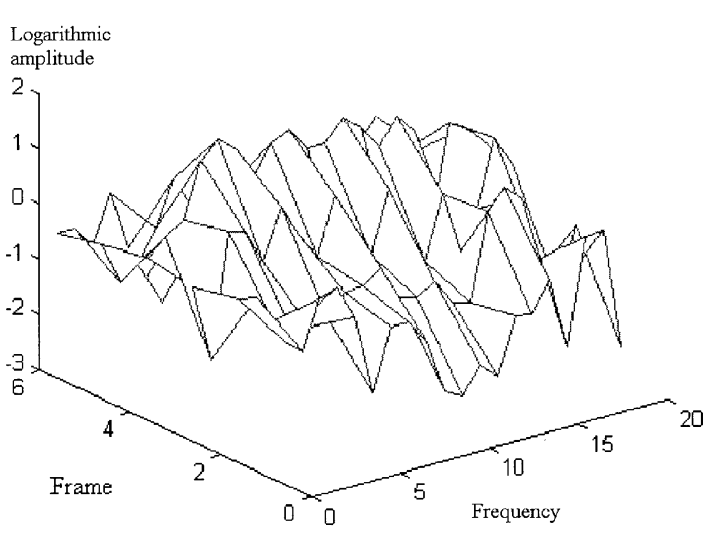

Fig. 4. The logarithmic spectrum of a signature.

In this paper, we use the logarithmic spectrum $\log |F F T(c)|$ to model the changing rate of the coordinates within the signature. With the principal component analysis based on the scatter matrices ${ }^{(8,9)}$ of the coefficients of logarithmic spectrum, only a small amount of coefficients are extracted to represent a signature. In this way, the amount of data is reduced because the number of extracted coefficients is much less than the number of coordinates in the signature.

\section{PRE-PROCESSING}

In pattern recognition, rotation and scaling may be handled during the matching process, such as relaxation matching, ${ }^{(10,11)}$ and a limited range of rotation angles can be tolerated. However, the complexity of matching would be greatly raised if rotation and scaling are considered exclusively in the matching stage. Hence, it would be more desirable that the problem of rotation and scaling can be resolved in the pre-processing stage before the matching procedures are taken.

Between rotation and scaling, rotation is more difficult to handle. "Which is the suitable reference point of the pattern?" If the reference point is itself rotationvariant, the rotation problem cannot be resolved. In this research, we propose that the center of the smallest enclosing circle (SEC) of the pattern could be selected as the reference point. The center of the SEC of the pattern is rotation-invariant.

The SEC of a set of points is obtained by finding the circumcircle of some three points of the set and by constructing the circle defined by some two points of the set as a diameter. ${ }^{(12,13)}$ In the following is the Algorithm 1: to obtain the SEC.

\section{Algorithm 1: Computing the SEC}

Input: The whole set of the points in the pattern. Output: The SEC of the pattern.

Step 1: Find out all the circumcircles of any three points of the pattern. Select the circumcircles which enclose the pattern to be the candidates of the SEC.
Step 2: Find out all the circles which use any two points of the pattern as the diameter. Select the circles which enclose the pattern to be the candidates of the SEC.

Step 3: From the candidate circles obtained from Steps 1 and 2, choose the circle whose radius is the smallest to be the SEC.

If we set the center of the SEC as the coordinate origin and normalize the $r$-coordinate to the radius of the SEC, then for the $(r, \theta)$ coordinates of the sample point $i$, say $\left(r_{i}, \theta_{i}\right), r_{i}$ is invariant to rotation and scaling. Also, $r_{i+1} / r_{i}$ and $\theta_{i+1}-\theta_{i}$ are invariant to rotation and scaling. If we combine the $x$ - and $y$ coordinates of the sample point $i$, say $\left(x_{i}, y_{i}\right)$, to obtain a complex value $x_{i}+j y_{i}$, then

$$
\frac{x_{i+1}+\mathrm{j} y_{i+1}}{X_{i}+\mathrm{j} y_{i}}=\frac{r_{i+1} \mathrm{e}^{\mathrm{j} \theta_{i+1}}}{r_{i} \mathrm{e}^{\mathrm{j} \theta_{i}}}=\frac{r_{i+1}}{r_{i}} \mathrm{e}^{\mathrm{j}\left(\theta_{i+1}-\theta_{i}\right)}
$$

is invariant to rotation and scaling. The computed result from adjacent $(x, y)$ coordinates in equation (1) is used for feature extraction.

\section{FEATURE EXTRACTION}

In speech processing, the whole set of speech sample points is divided into small frames of sample points. This helps to preserve "short time stationary" for spectral analysis. The spectral analysis is then taken for each speech frame. The changing rate of coordinates in the signature waveform is not so wild as that in the speech waveform. Therefore, we just divide the sample points in each word of the signature into smaller number of frames.

The steps of extracting dynamic features of a signature are shown in Fig. 5 and the details are described as follows:

Step 1: Resample the signature word and divide the sample points into frames. We resample every word in the signature into a fixed number of sample points by interpolation to overcome the difference in sampling rates for different writing tablets. We define FR to be the desired number of frames in a signature word, FRAME_SIZE the size of each frame and FRAME_SHIFT the number of sample points shift between two adjacent frames. Thus, the total number of sample points after resampling is FRAME_SIZE + (FR - 1) FRAME_SHIFT. There are FRAME_SIZE - FRAME_SHIFT overlapped sample points between two adjacent frames. If the time duration for writing the signature word is $T$, then the signature word is resampled every $T /[$ FRAME_SIZE $+($ FR -1$) \cdot$ FRAME_SHIFT $]$ time units.

Step 2: Combine the $x$-coordinates and the $y$-coordinates to produce a complex series: ${ }^{(14)}$

$$
c(n)=\frac{x(n)+\mathrm{j} y(n)}{x(n-1)+\mathrm{j} y(n-1)} .
$$




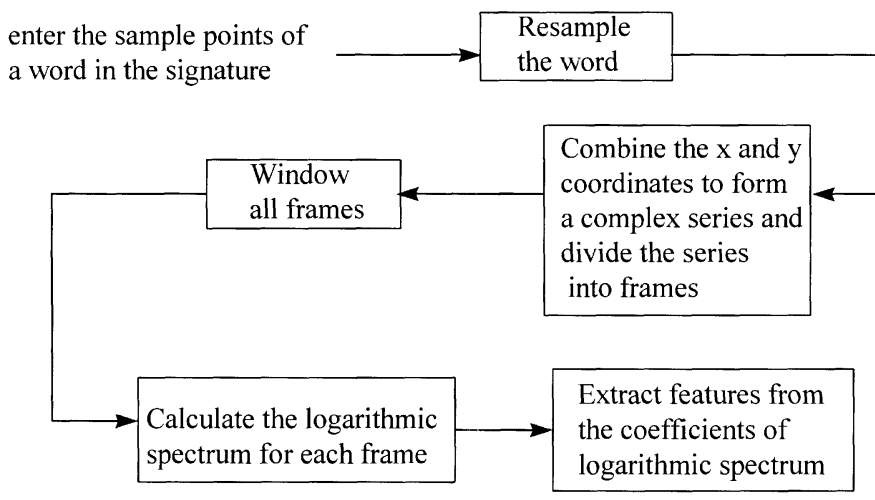

Fig. 5. The steps for extracting dynamic features.

According to the description in Section 2, $c(n)$ is invariant to rotation and scaling.

Step 3: Window each individual frame so as to minimize the discontinuities at the beginning and the end of each frame. We apply the Hamming window function $w(n)^{(4,5)}$ to each frame of sample points, $c_{f}=\{c(n)\}$. The result of windowing is $c_{f}^{*}=\left\{c^{*}(n)\right\}$ where

$$
c^{*}(n)=c(n) w(n)
$$

and

$$
w(n)=0.54-0.46 \cos \left(\frac{2 n \pi}{F R A M E_{-} S I Z E-1}\right) .
$$

Step 4: Calculate the logarithmic spectrum for each frame:

$$
C=\log \left|F F T\left(c_{f}^{*}\right)\right| .
$$

Therefore, there are $F R \cdot F R A M E \_S I Z E$ coefficients for a word in the signature.

Step 5: Extract features from the logarithmic spectrum: In order to reduce the amount of data for featuring signatures, we apply the intra-category and inter-category scatter matrices ${ }^{(8)}$ to the feature extraction of our signature verification scheme. In this research, the intra-category scatter matrices are replaced by intra-frame scatter matrices, while the inter-category scatter matrices are replaced by interframe scatter matrices. An intra-frame scatter matrix shows the scatter of the coefficients of logarithmic spectrum around the expected coefficients of their respective frames, and is expressed by

$$
\begin{aligned}
S C A_{w} & =\frac{1}{F R} \sum_{i=1}^{F R} E\left\{\left(C-M_{i}\right)\left(C-M_{i}\right)^{\mathrm{T}}\right\} \\
& =\frac{1}{F R} \sum_{i=1}^{F R} \Omega_{i},
\end{aligned}
$$

where $\Omega_{i}$ is the covariance matrix of the coefficients of the $i$ th frame, and $M_{i}$ is the expected vector of that frame. An inter-frame scatter matrix is the scatter of the expected coefficients around the mixture mean $M_{0}$ and is expressed as

$$
S C A_{b}=\frac{1}{F R} \sum_{i=1}^{F R}\left(M_{i}-M_{0}\right)\left(M_{i}-M_{0}\right)^{\mathrm{T}},
$$

where

$$
M_{0}=\frac{1}{F R} \sum_{i=1}^{F R} M_{i}
$$

By maximizing the trace of $S C A_{w}^{-1} \cdot S C A_{b}$, i.e. $\operatorname{tr}\left(S C A_{w}^{-1} \cdot S C A_{b}\right)$, we can extract features with maximum frames separability and thus make the transitions among frames as obvious as possible. The matrix of eigen vectors corresponding to the $\mathrm{N}$ largest eigen values of $S C A_{w}^{-1} \cdot S C A_{b}$ are calculated as

$$
\Phi=\left[\begin{array}{lll}
\phi_{1} \phi_{2} & \ldots & \phi_{N}
\end{array}\right]
$$

where each $\phi_{i}$ is the $i$ th eigenvector in column form. Then we may extract $N$ values from FRAME_SIZE coefficients, $C$, of a frame by calculating

$$
C^{*}=\Phi^{\mathrm{T}} \cdot C,
$$

where $C^{*}$ and $C$ are all column vectors. The number of elements in $C$ is FRAME_SIZE, while the number of elements in $C^{*}$ is $N$. From the properties of the scatter matrices, ${ }^{(8)} S C A_{b}$ has rank FR-1 and $S C A_{w}$ has full rank. Therefore, the rank of $S C A_{w}^{-1} \cdot S C A_{b}$ is FR-1. We set $N$ to be FR-1.

A signature could be regarded as consisting of several words, depending on the skills used for signature segmentation. The easiest way is to regard the whole signature as a big word no matter how the signature is signed. Another convenient approach is to restrict the users to write signature words in different locations on the tablet to avoid segmentation problem. In the case that a signature contains $M$ separate words, there will be coefficients to feature the whole signature. Typically, $M$ is 3 for Chinese signatures.

\section{DYNAMIC ANALYSIS OF ON-LINE SIGNATURES BASED ON LOGARITHMIC SPECTRUM}

In this section, the dynamic analysis of on-line signature verification using logarithmic spectrum as the changing rate of coordinates is described. The similarity between two sets of coefficients of logarithmic spectrum, say $\mathbf{V}$ and $\mathbf{W}$, can be computed using 
the similarity measurement formula (11). We assume that sequences $\mathbf{V}$ and $\mathbf{W}$ are constructed by frames $V_{0}, V_{1}, \ldots, V_{p-1}$ and frames $W_{0}, W_{1}, \ldots, W_{p-1}$ with each frame being a vector $V_{i}=\left(v_{i}^{0}, v_{i}^{1}, \ldots, v_{i}^{q-1}\right)$, and $W_{i}=\left(w_{i}^{0}, w_{i}^{1}, \ldots, w_{i}^{q-1}\right)$, respectively. $p$ is the number of frames. $q$ is the feature dimension of $V_{i}$ and $W_{i}$, $0 \leq i \leq p-1$. The similarities of coefficients of logarithmic spectrum between corresponding words of the two signatures are computed. Then the dynamic similarity of all words are combined to derive the dynamic similarity between two signatures by equation (12).

$$
\begin{aligned}
& \operatorname{Similarity}(V, W) \\
& =0.5+\frac{\sum_{i=0}^{p-1} \sum_{j=0}^{q-1} v_{i}^{j} \cdot w_{i}^{j}}{\sum_{i=0}^{p-1} \sum_{j=0}^{q-1}\left(v_{i}^{j} \cdot v_{i}^{j}+w_{i}^{j} \cdot w_{i}^{j}\right)} . \\
& S=\frac{1}{M} \sum_{i=1}^{M} S_{i} .
\end{aligned}
$$

The reference templates and the verification thresholds of registered persons must be computed before the verification system can determine the genuineness of the input signature. For each registered person, we use the mean values of the corresponding coefficients of logarithmic spectrum as the reference template. Listed in the following is the formula for the computation of the verification threshold of dynamic analysis:

$$
\begin{gathered}
T_{d} \text { median }\left(D Y S_{j}\right)-\min (0.12, \max (0.06,2.5 \\
\left.\left.\times\left(\max _{j}\left(D Y S_{j}\right)-\underset{j}{\operatorname{median}}\left(D Y S_{j}\right)\right)\right)\right),
\end{gathered}
$$

where $T_{d}$ is the threshold of dynamic similarity, $D Y S_{j}$ is the dynamic similarity between the $j$ th training signature and the reference template.

In the verification, the reference template of the registered person claimed by the user is picked up. Then we use it to calculate the dynamic similarity between input signature and the reference template. If the similarity is larger than the verification threshold of the dynamic similarity, the input signature passes the testing of dynamic analysis and is regarded as a genuine one.

\section{EXPERIMENTATION}

We collected genuine signatures from 27 registered persons. Each signature contains three words. Each registered person signed his/her signatures for about 30 times, ten of which are used to acquire the reference templates and the verification thresholds and others are used for the testing. There are in total 560 genuine signatures of all registered persons for system testing. The imitators totally forged 650 signatures. The forged signatures were imitated by four "expert" imitators. The genuine signatures were shown to the imitators for repeating practice for a period of time before actual collection of forgery data for experimentation. Some examples of genuine and forged signatures for the experimentation are shown in Fig. 6.

For the genuine signatures, we recorded the timing of the strokes within each signature word and the timing of the sample points within each stroke. The genuine signatures were shown to the signees according to their timing information. Before signing signatures, each genuine user or imitator input the ID number and the system used this ID number as the key to retrieve one of the previously signed genuine signatures. This previous signature was shown to him/her when he/she signed.

We used an OmniPen OP-1000 digitizer to capture the signature trajectories. The data rate is 200 reports per second. The spatial resolution is 1016 lines per inch. The related data of the experimentation are listed in Table 1.

False rejection rate is defined to be the rate that the genuine signatures are classified as forgeries. False rejection error is also called Type I error. False acceptance rate is the rate that the forgeries are classified as genuine ones. False acceptance error is also called Type II error. The mixed error rate is defined to be the ratio of the number of mis-verified genuine and forged

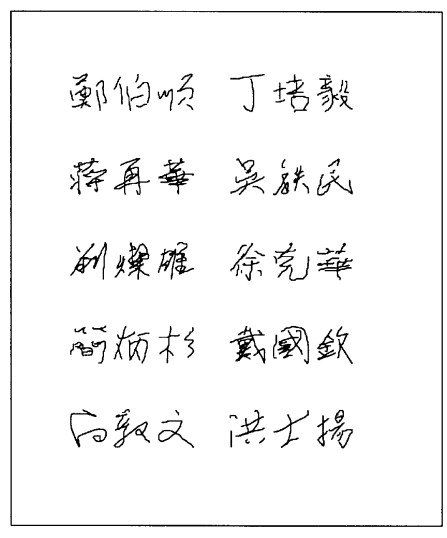

(a) genuine signatures

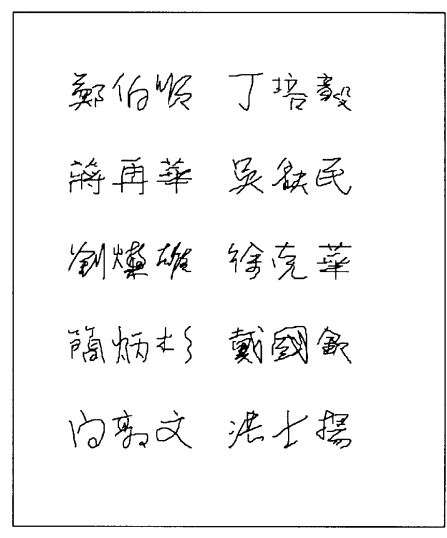

(b) forged signatures

Fig. 6. Examples of genuine and forged signatures of the experimentation. 
Table 1. Legends of the experimentation

\section{Number of registered persons}

Total number of training signatures

Total number of genuine signatures for testing

Total number of forged signatures from 4 imitators

Total number of sample points in a signature word,

SAMPLES = FRAME_SIZE + FRAME_SHIFT*(FR-1)

The number of frames in a word (FR)

The number of sample points shift between two adjacent frames (FRAME_SHIFT)

The number of sample points in a frame (FRAME_SIZE)

The number of words in a signature $(\mathrm{M})$

The number of extracted coefficients of logarithmic spectrum of a frame (N)
27

270 (10 signatures from each person)

560

650

100

Varies

The integer part of (SAMPLES/FR)

SAMPLES- FRAME_SHIFT*(FR-1) 3

FR-1

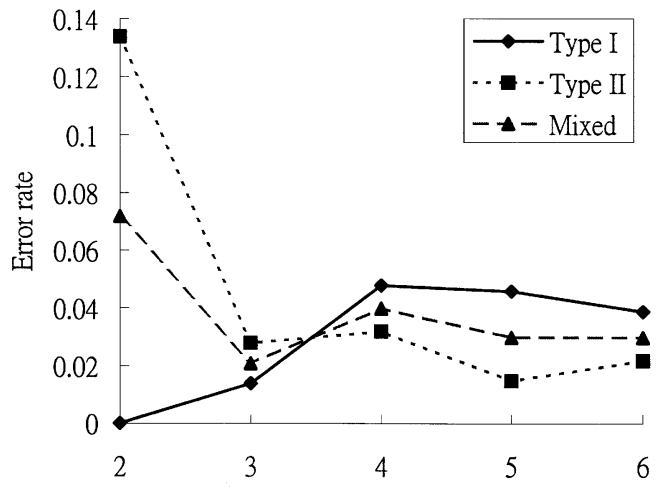

FR, the number of frames in a signature word

Fig. 7. Error rates of dynamic analysis with the number of frames in a signature word being variable.

signatures over the total number of genuine and forged signatures. Thus, if false rejection and false acceptance rates are $A / 560$ and $B / 650$, then the mixed error rate should be $(A+B) /(560+650)$.

Figure 7 shows Type I, Type II and the mixed error rates of verification using dynamic analysis with the number of frames in a signature word being variable. According to the discussion of the feature extraction of logarithmic spectrum using principal component analysis, the number of extracted coefficients of logarithmic spectrum of a frame is FR-1. Therefore, there are $F R \cdot(F R-1)$ coefficients extracted to characterize the dynamics of a signature word. From the experimental result shown in Fig. 7, setting the number of frames in a signature word to be three seems the most suitable in this experimentation based on the collected data set. There are only six coefficients to feature the dynamics of a signature word in this case.

From the experimentation, most signatures are verified with high accuracy. However, as the writing behaviors of some persons are unstable and timevariant, a genuine signature written at time instance $t_{1}$ may differ greatly from the one which is written at time instance $t_{2}$ with or without intention. Therefore, the reference templates should be frequently updated in order to catch up with the recent writing behaviors of the registered persons.
Other factors to affect the quality of signatures include the quality of the writing tablet and the mood of the writer. Most of the errors occur because signing on the tablet is not so smooth as signing on the paper. Under such circumstances, extra variability is imposed into signatures. If this extra variability appears in the training set, the verification threshold may be reduced and the Type II error rate increases. Even this extra variability does not appear in the training set to affect the verification threshold, it may also appear in the test set to make genuine signatures look like forgeries. Under such circumstances, the Type I error rate increases. Therefore, the equipped electronic pen/tablet to the signature verification system must be as smooth to use as possible.

Because the signees know that the signature collection is only for experimentation, they cannot be as serious as they are signing a check for withdrawing money. Fortunately, we may expect the signees to be more serious in signing their signatures when they are making real commercial transactions. Hence, the Type I error rate may be lower in signature verification for real commercial transactions.

\section{CONCLUSIONS}

In this paper, the dynamic analysis of on-line signature verification using logarithmic spectrum as the changing rate of the coordinates is proposed. Extracted coefficients based on scatter matrices provide useful features for characterizing a signature. The mean values of the corresponding coefficients calculated from corresponding training signatures are used as reference template for each registered person. The similarity of the changing rate of coordinates between two signatures can be presented by computing the similarity between their corresponding set of extracted coefficients of logarithmic spectrum. In the verification, the reference template of the registered person claimed by the user is picked up. Then we use it to calculate the dynamic similarity between input signature and the reference template. If the similarity is larger than the verification threshold of the dynamic similarity, the input signature passes the testing of dynamic analysis and is regarded as a genuine one. 
From performance tests on 27 registered persons, the Type I and Type II error rates are as low as $1.4 \%$ and $2.8 \%$, respectively. The effectiveness of the proposed scheme is thus demonstrated.

\section{REFERENCES}

1. R. Plamondon and G. Lorette, Automatic signature verification and writer identification - the state of the art, Pattern Recognition 22(2), 107-131 (1989).

2. C. F. Lam and D. Kamins, Signature recognition through spectral analysis, Pattern Recognition 22(1), 39-44 (1989).

3. J. D. Markel and A. H. Gray, Jr, Linear Prediction of Speech, Springer, Berlin (1980)

4. S. Furui, Digital Speech Processing, Synthesis, and Recognition, Marcel Dekker, Inc., New York (1989).

5. L. Rabiner and B.-H. Juang, Fundamentals of Speech Recognition, Prentice-Hall International, Inc. Englewood Cliffs (1993).

6. Q.-Z. Wu, I.-C. Jou, B.-S. Jeng, N.-J. Cheng, S.-S. Huang, P.-Y. Ting, D.-M. Shieh and C.-J. Wen, On-line signature verification using neural networks, Proc. Int. Symp. on Artificial Neural Networks, Taiwan, R. O. C., pp. 478-482, 15-17 December (1994).
7. Q.-Z. Wu, I.-C. Jou and S.-Y. Lee, On-line signature verification using LPC cepstrum and neural networks, IEEE Trans. Systems Man Cybernet. 27(1), 148-153 (1997).

8. Keinosuke Fukunaga, Introduction to Statistical Pattern Recognition, Second Edition, Academic Press, New York (1990).

9. R. C. Gonzalez and R. E. Woods, Digital Image Processing, Addison-Wesley Reading, Massachusetts (1993).

10. L.-H. Chen and J.-R. Lieh, Handwritten character recognition using a 2-layer random graph model by relaxation model, Pattern Recognition 23(11), 1189-1205 (1990).

11. L. Lam and C. Y. Suen, Structural classification and relaxation matching of totally unconstrained handwritten zip-code numbers, Pattern Recognition 21(1), 19-31 (1988).

12. H. Rademacher and O. Toeplitz, The Enjoyment of Mathematics, Princeton University Press, Princeton, NJ (1957).

13. F. P. Preparata and M. I. Schamos, Computational Geometry: An Introduction, Springer, New York (1985).

14. Y. Sato and K. Kogure, On-line signature verification based on shape, motion and handwriting pressure, Proc. 6th Int. Conf. On Pattern Recognition, Munich, Vol. 2, pp. 823-826 (1982).

\begin{abstract}
About the Author-QUEN-ZONG WU received his B.S. degree from the Department of Electrical Engineering, Tatung Institute of Technology, Taiwan, in 1987, his M.S. degree in computer science and information engineering from National Chiao Tung University, Taiwan, in 1989, and his Ph.D. degree from the Department of Computer Science and Information Engineering, National Chiao Tung University, Taiwan, in 1997. Presently, Dr. Wu is with Chunghwa Telecommunication Laboratories, Taiwan. His current research interests include pattern recognition, image processing, and neural networks.
\end{abstract}

\begin{abstract}
About the Author-SUH-YIN LEE received her B.S.E.E. degree from the National Chiao Tung University, Taiwan, in 1972, and her M.S. degree in computer science from the University of Washington, Seattle, in 1975. She joined the faculty of the Department of Computer Engineering at Chiao Tung University in 1976 and received the $\mathrm{Ph} . \mathrm{D}$. degree in electronic engineering there in 1982. Dr. Lee is now a professor in the Department of Computer Science and Information Engineering at Chiao Tung University. She chaired the department from 1991 to 1993 . Her current research interests include multimedia information systems, object-oriented databases, image/spatial database, and computer network. Dr. Lee is a member of Phi Tau Phi, the ACM, and the IEEE Computer Society.
\end{abstract}

About the Author-I-CHANG JOU received his B.S. degree from the Electrical Engineering Department, National Taiwan University, Taiwan, in 1969, the M.S. degree in geographics and computer science from National Central University, Taiwan, in 1972 and 1983, respectively, and the Ph.D. degree from the Electrical Engineering Department, National Taiwan University, Taiwan, in 1986. Presently, he chairs the Electrical Engineering Department, Chang Gang University, Taiwan. His current research interests include VLSI for DSP, digital signal processing, image processing, speech processing, and neural networks. He is a senior member of IEEE. 